\title{
STELLAR MIXING FROM GALACTIC AND MAGELLANIC CLOUD PLANETARY NEBULAE ABUNDANCES
}

\author{
R. E. S. CLEGG \\ Royal Greenwich Observatory \\ Madingley Road \\ Cambridge CB3 0EZ, UK.
}

\begin{abstract}
The mixing and nucleosynthetic processes which occur in the main-sequence and red giant stages of evolution and which can affect measured PN abundances are discussed. It is suggested that samples of planetary nebulae contain a sufficient range of progenitor stars' initial metallicities, angular momenta, binarity, etc. so as to explain the large scatter always seen in abundance diagrams for PN samples. CNO abundances in the Galactic disk and in the Magellanic Clouds are reviewed. New results for nebular abundances and central star properties in the Clouds are given. The current red giant population in the Clouds mix out more ${ }^{12} \mathrm{C}$ per star than their Galactic disk counterparts.
\end{abstract}

\section{Introduction}

Virtually all low (1-3 $\left.\mathrm{M}_{\odot}\right)$ and intermediate-mass $\left(3-8 \mathrm{M}_{\odot}\right)$ stars are believed to go through a planetary nebula (PN) phase, whether as single stars or as members of multiple systems. Some stars which are in binary systems may avoid this fate by having their ejected mass captured by a companion, either in the form of a 'stored matter' in an accretion disk or deposited directly onto the secondary star's surface. But, even in this case, such ejecta will probably appear as part of a planetary nebula once the secondary star itself has evolved through the Asymptotic Giant Branch (AGB) stage.

Analysis of PN abundances gives information on the sum of all the mixing processes that there have been during the pre-main sequence, main-sequence, first red giant branch and asymptotic red giant branch stages of evolution of these stars. Also, the PNs provide information about the elements $\mathrm{He}, \mathrm{Ne}, \mathrm{Ar}$ and $\mathrm{S}$ (which cannot usually be measured in red giant spectra), as well as the important $\mathrm{CNO}$ group. PNs are enriched in $\mathrm{He}, \mathrm{C}$ and $\mathrm{N}$ (some possibly also in $\mathrm{Ne}$ ), and measured abundances then show the contribution of these stars to the chemical evolution of the Galaxy, which is especially important for carbon. Finally, PN abundances can be obtained in the Galactic disk, Galactic Bulge, Magellanic Clouds and even some Local Group galaxies, enabling stellar evolution in sites of differing metallicities and evolutionary histories to be studied quantitatively. These reasons show the importance of nebular abundance studies.

Recent reviews on PNs and their abundances have been given by Peimbert (1990), Henry (1990), Clegg (1989, 1990), and Kaler (1985). 


\section{Current Studies and Problems}

\subsection{Background and Commentary}

The processes which alter surface abundances, all the way from pre-main sequence stage to PN stage, are nucleosynthesis and mixing. The relevant nuclear burning includes $\mathrm{CN}$ and NO-cycle H-burning, which alters isotopic abundances of $\mathrm{C}, \mathrm{N}, \mathrm{O}$ and $\mathrm{F}$; helium-burning, which produces chiefly $\mathrm{C}$ with lesser amounts of $\mathrm{O}$ and $\mathrm{Ne}$; and related reactions in the burning zones of luminous red giants in which for example ${ }^{14} \mathrm{~N}$ may be mixed into a $\mathrm{He}$ burning zone, or neutron capture onto ${ }^{13} \mathrm{C}$ or ${ }^{22} \mathrm{Ne}$ may produce both enhancements of heavy-elements and altered isotopic mixtures of lighter elements such as $\mathrm{Ne}, \mathrm{Mg}$ and $\mathrm{Ti}$. The mixing processes include convection/semi-convection, meridional circulation currents, diffusion, gravitational settling and radiative acceleration on specific ions. Material processed inside the star can get picked-up in one of three 'dredge-up' phases described by Iben \& Renzini (1983). Mixing 'extra' to this is provided by meridional circulation currents (Sweigert \& Mengel 1979) and by a (very deep) surface convection zone which for massive red giants may extend down to regions hot enough for $\mathrm{CN}$-cycle reactions to process the stellar envelope directly (rather than in a delayed pick-up which the 3 dredge-up stages are).

Peimbert (1978) usefully assigned PNs to four classes. Type I have high He and/or N abundances, are usually strongly bi-polar in shape, and are thought to be the progeny of stars in the mass range about $2.5-8 \mathrm{M}_{\odot}$. Types II and III are Galactic disk nebulae with low and high velocity dispersions, respectively, perpendicular to the Galactic plane; and Type IV are extreme halo objects (such as K648, the nebula in the globular cluster M15). The sequence is thus roughly one of decreasing initial mass and increasing age.

Four 'classic' papers which give theoretical descriptions of the mixing processes which affect PN abundances are by Iben \& Truran (1978) on thermally-pulsing stars, Becker \& Iben (1979 and 1980) on the results of the 1st, 2nd \& 3rd dredge-up episodes for intermediatemass stars (3-8 $\left.\mathrm{M}_{\odot}\right)$; and finally by Sweigart \& Mengel (1979) on the effects of meridional circulation currents. In fact, a typical sample of nearby Galactic PNs will contain mostly lower mass stars than this, since the average mass of disk stars evolving now will be approximately $1.5 \mathrm{M}_{\odot}$ (e.g., Scalo 1981). For low-mass stars, recent evolutionary calculations have been given by Sackmann \& Boothroyd (these proceedings), Lattanzio (1989) and Hollowell \& Iben (1989).

It is a prediction of the calculations of Becker \& Iben for 3-8 $\mathrm{M}_{\odot}$ stars that enhancements in surface He, C \& N are larger at low metallicity ' $z$ '; and Sweigart \& Mengel too predict that the effect of circulation currents (mainly, higher $\mathrm{N}$ abundances) are greater at low $\mathrm{z}$. For lower mass models, Wood (1981) found that the dredge-up of ${ }^{12} \mathrm{C}$ occurred in lower AGB core masses at lower metallicities. This effect (see Sec. 4) could enhance production of $\mathrm{C}$ in a metal-poor population (e.g. in the Magellanic Clouds).

Another prediction (Becker \& Iben, Iben \& Renzini 1983) is that O/H hardly changes from the main sequence to PN stage. I compare mean values, in units of $10^{-4}$, for nearby disk samples: Galactic PNs, H II regions: $4.4( \pm 0.5)$; M and S giants: $6.3( \pm 1.8)$ (Smith \& Lambert 1990); cool carbon stars: 4.7( \pm 1.5$)$ (Lambert et al. 1986); Solar photosphere: $8.7( \pm 1.2)(\mathrm{N}$. Grevesse, these proceedings). The result for PNs \& H II regions agrees, 
within the errors, with the value for red giants, but is significantly different from the Solar value. I assume that this is due to the 'metallicity of the Sun'; one can remove metallicity effects where necessary by working with ratios to oxygen (e.g., $\mathrm{Ne} / \mathrm{O}$, etc).

A difficulty in the detailed interpretation of PN abundances is that, as noted above, such a wide range of stars pass through this 'box' on the HR diagram. This means that a spread of initial metallicities, rotational velocity histories, binarity status, ... enter the 'box', and my thesis in this article is to suggest that it is this that causes the observed spreads (sometimes seen as scatter diagrams) in nebular abundance plots. A good example to quote here is the nitrogen abundance in PNs. It is affected by CN and ON-cycle processing, and $\mathrm{N}$ gets mixed out in the 1st, 2nd \& perhaps even the 3rd 'dredge-up' episodes during red giant evolution (see Iben \& Renzini 1983). But CNO abundance studies of Pop. I and Globular cluster stars (e.g., Smith 1987; C. Sneden, these proceedings) show that there is often 'more' mixing than predicted theoretically, betrayed especially by high ${ }^{13} \mathrm{C}$ and ${ }^{14} \mathrm{~N}$ abundances. Probably, 'extra' processing has ocurred both on the main sequence and on the red giant branch, with meridional circulation currents the chief suspect.

Our awareness of the role of binary mass-transfer has grown recently. A considerable fraction of the peculiar red giants do not contain Tc, and these, like the BaII stars, are interpreted as having received processed material from a (now unseen) companion. Eventually their envelope will be polluted again (from within), and the subsequent ejected nebula will have a mix of CNO, etc. not corresponding to any single-star calculation. [The large fraction of binaries must also have a strong role in the 'shaping' of PNs, most of which are bipolar to some degree].

\subsection{Current Problems in Abundance Determinations}

1. CNO in dust grains? This would be important for $\mathrm{C}$ and $\mathrm{O}$, but probably they are not depleted by more than $30 \%$. The evidence includes: low dust/gas ratios derived from detailed models matching IRAS and ground-based IR data (Hoare 1990, Hoare \& Clegg 1988, Harrington, Monk \& Clegg 1988) for O-rich and C-rich nebulae; consideration of the available supply of $\mathrm{Mg}$, Si \& $\mathrm{Al}$ to form, with oxygen, the silicate material which is observed at $9.7 \mu \mathrm{m}$; and the observed modest depletion of $O$ in diffuse clouds (Jenkins 1987) - especially relative to the Orion $O$ abundance.

2. Temperature fluctuations (Peimbert 1967, Rubin 1969, Dinerstein et al. 1985). If these exist at a significant level, 'true' $\mathrm{O} / \mathrm{H}$ values could be up to 0.15 dex higher than 'apparent', but ratios like $\mathrm{N} / \mathrm{O}$ and $\mathrm{Ne} / \mathrm{O}$ are much less affected.

3. Non-linear detectors. It is known now that the IDS can be non-linear (see discussion by Peimbert \& Torres-Peimbert 1987a), as can the IPCS sometimes.

4. Carbon Abundances. A current problem is that derived values of $\mathrm{C}^{++} / \mathrm{H}^{+}$from a recombination line (C II 4267 $\AA$ ) and a collisionally-excited line (C III 1908 $\AA$ ) disagree systematically by up to a factor 4 (Kaler 1986; Barker 1987 and references therein).

5. Assumption of uniform abundances. For 3 cases - A30, A58 and A78 - we know there are $\mathrm{He}-$ and $\mathrm{C}$-rich knots without $\mathrm{H}$ around the central stars (the 'born-again' nebulae interpreted by Iben et al. 1983). Are there other C-rich cores (if so this could explain the above problem)? One idea is simply that the red giant envelope is more C-rich towards the end of the ejection of $\sim 0.2 \mathrm{M}_{\odot}$ of envelope, though no theory currently 
predicts this. Another idea is that a C-rich wind 'injects' carbon. For example, a WC-type nucleus with $\mathrm{C} / \mathrm{He} \approx 0.2$ and $\dot{\mathrm{M}} \sim 10^{-8} \mathrm{M}_{\odot} \mathrm{yr}^{-1}$ ejects about $4 \times 10^{-5} \mathrm{M}_{\odot}$ of $\mathrm{C}$ in $10^{4} \mathrm{yr}$. The surrounding nebula with $0.2 \mathrm{M}_{\odot}$ and $\mathrm{C} / \mathrm{H} \approx 10^{-3}$ contains $2 \times 10^{-4} \mathrm{M}_{\odot}$ of carbon, so a $\mathrm{C}$-rich wind would contribute mostly just to the carbon recombination lines from the (cooler) nebular centre.

6. Collisional excitation of He I lines. Peimbert \& Torres-Peimbert (1987a,b) found there may only be about $60 \%$ as much metastable $\left(2 \mathrm{~s}{ }^{3} \mathrm{~S}\right) \mathrm{He}^{0}$ in PNs as predicted (Clegg 1987). This uncertainty has a small effect on Galactic nebulae, but a significant one on LMC \& SMC PNs (the correction needed rises with electron temperature \& density, and temperatures are higher in Cloud PN due to low abundances there).

\section{Recent Studies and Comparison with Theory}

\subsection{Galactic and Magellanic Cloud Nebulae}

Recent reviews of PN abundances (Peimbert 1990, Clegg 1989) will not be repeated here. In summary, $\mathrm{PNs}$ are overabundant in helium $(\mathrm{He} / \mathrm{H}$ values $0.10-0.20$ but mostly around 0.10, c.f. H II regions value of 0.10 ); carbon (attributed to the 3 rd dredge-up of ${ }^{12} \mathrm{C}$ ); nitrogen (the 1st dredge-up for all stars, the 2nd for $\mathrm{M}>2.5 \mathrm{M}_{\odot}$, and possibly 'envelope burning' of fresh $\mathrm{C}$ after the 3rd dredge-up; plus 'extra' $\mathrm{N}$ from circulation currents and the CN-cycle); and possibly in neon (Type I PN only; marginal evidence).

Based mainly on the results of Becker \& Iben (1979, 1980), Renzini \& Voli (1981; RV) computed expected surface abundances of CNO expected after the 1st, 2nd and 3rd dredge-up episodes. Several authors since then have compared PN abundance plots with RV predictions, both for the Galaxy and the Magellanic Clouds. Recent published comparisons include those by Kaler \& Jacoby (1989, 1990); Kaler, Shaw \& Kwitter (1990); Henry, Liebert \& Boroson (1990); Henry (1990); and Stasinska \& Tylenda (1990).

The result is always the same. There is a large scatter about the RV predictions (e.g., my Figures 1 \& 2). I suggest much of it is real. There are at least 3 reasons why the data will not agree with the 'simple' RV predictions: (i) some of the input calculations are for 3-8 $\mathrm{M}_{\odot}$ stars, but most Disk PNs arise from $\mathrm{M} \leq 2.5 \mathrm{M}_{\odot}$ stars; (ii) we now know that many AGB stars have large mass-loss rates and simply do not live long enough on the AGB for all the 'predicted' processes to occur; (iii) a mix of stellar abundances, rotation velocities, binarity, etc. is passing through the 'PN Box' now, as noted earlier.

Nevertheless, observed nebular abundances can still be interpreted qualitatively in terms of known processes such as convective dredge-up, circulation currents, and transfer of matter from a (more massive) companion.

Several current disagreements between 'simple theory' and observation are noted. Kaler et al. (1990) found very high $\mathrm{He} / \mathrm{H}$ ratios (0.20-0.26) and $\mathrm{N} / \mathrm{O}$ ratios (0.8-2.0) in some large, evolved nebulae. Explanation of such values demands 'extra' mixing, which can probably be provided by meridional currents operating over long times in the main sequence and red giant stages. Secondly, Kaler \& Jacoby, and Kaler et al., find that in plots of N/O versus core-mass or stellar temperature, the $\mathrm{N} / \mathrm{O}$ ratio rises much more steeply than predicted: another example of excess $\mathrm{N}$ being mixed up. Also in some Type I nebulae, high $\mathrm{N} / \mathrm{O}$ 


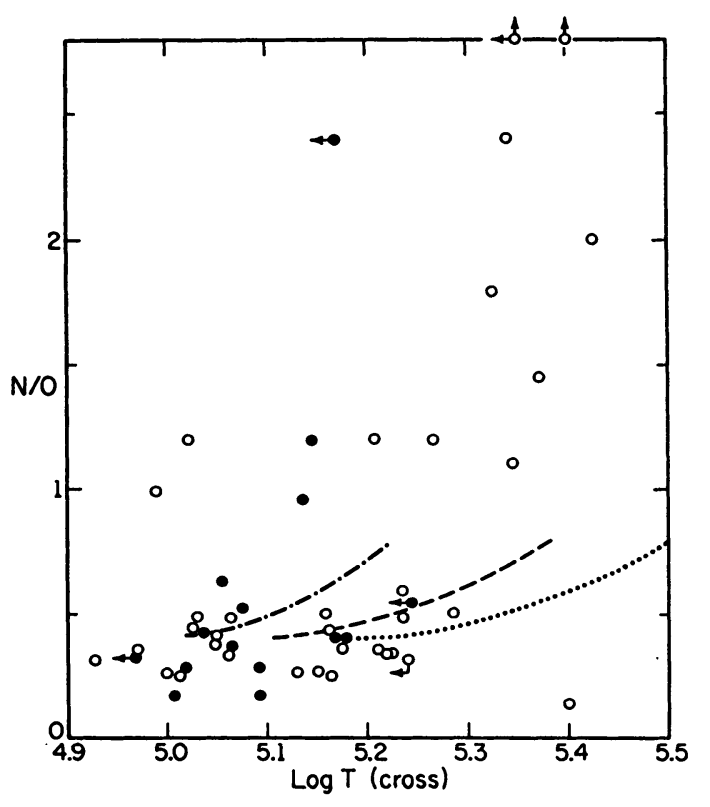

F1g. 1. (Kaler \& Jacoby 1989). N/O versus Central Star temperature. Curves- theoretical predictions.

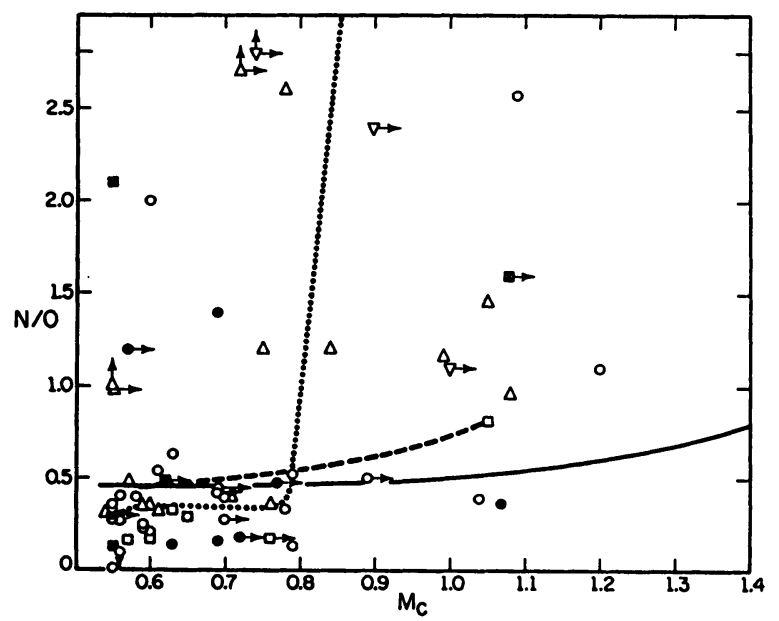

Fig.2. (Kaler et al.1990). N/O versus core mass. Note the large scatter in both these diagrams. 
and low $\mathrm{O} / \mathrm{H}$ ratios are seen (e.g., for $\mathrm{Hu1}-2, \mathrm{~N} / \mathrm{O}=1.4$ and $\mathrm{O} / \mathrm{H}=1.6 \times 10^{-4}$ ), with an anti-correlation between $\mathrm{N} / \mathrm{O}$ and $\mathrm{O} / \mathrm{H}$ (e.g., Henry 1990; Kaler et al.). As such objects are thought to derive from 3-8 $\mathrm{M}_{\odot}$ stars and many are located closer to the Galactic centre than is the Sun, this is taken as evidence for ON cycle H-burning with extent greater than predicted by the Becker \& Iben/RV calculations.

In fact, the $\mathrm{N} / \mathrm{O}-\mathrm{O} / \mathrm{H}$ anti-correlation was found to be very significant for Magellanic Cloud nebulae by Henry et al. (1989), and quite significant by Kaler al. for large, local PNs. This contrasts with a marginal relationship for local, disk PNs in other studies e.g., Henry 1990). Henry et al. conclude that there is an optimum metallicity and red giant mass for envelope processing through the $\mathrm{ON}$-cycle: for example, the LMC has low metallicity and probably many 2-3 $\mathrm{M}_{\odot}$ stars evolving now, due to recent 'starbursts'.

Smith \& Lambert (1990) compared Galactic PN and red giant CNO abundances. The mean $\mathrm{O} / \mathrm{H}$ values agree within the errors, for $\mathrm{M}, \mathrm{S}, \& \mathrm{~N}$ stars on the one hand and for the planetaries on the other. However, present red giant abundances are for non-Mira variables (for good reason), and we do not yet fully understand the evolutionary sequence involving semi-regular red giants, Mira variables, post-AGB stars, and planetaries. The biggest discordance is found in comparing the nitrogen abundances of $\mathrm{O}$-rich and $\mathrm{C}$-rich giants with the same classes of PNs. The $C$ stars all have low $N$ abundances $(\log A \leq 8.1$, Lambert et al. 1986) and are clearly separated from the $O$-rich $M$ and $S$ stars $(\log A \geq 8.1)$. But for $\mathrm{PNs}$, no such correlation is seen between $\mathrm{C} / \mathrm{O}$ and $\mathrm{N} / \mathrm{H}$ ratios. Either the $\mathrm{N}$ abundances of the carbon stars are in error, or (more interesting) the continuing evolution of semi-regular carbon stars - perhaps through a Mira phase - produces some much higher $\mathrm{N}$ abundances that 'fill in' the apparent gap at the high $\mathrm{N} / \mathrm{H}$ end.

\subsection{Abundances in Giant Halos}

A recent discovery that bears on the mixing inside AGB stars is the result that three nebulae with giant ionized 'halos', which have an expansion age of $10^{5} \mathrm{yr}$ rather than the main nebula's $10^{4} \mathrm{yr}$, have high $\mathrm{T}_{e}$ values and apparently-low abundances of $\mathrm{N}, \mathrm{O}$ and Ne (Middlemass, Clegg \& Walsh 1989; Manchado \& Pottasch 1989). One might suppose that the abundance results are real and the high temperatures are simply due to the low abundances. But the first authors found that this did not work quantitatively. A reason to be suspicious is that all three of $\mathrm{N}, \mathrm{O}$ and Ne seem to be deficient by just a factor two in the halos. This led us (Middlemass et al. 1990) to develop a model in which a fast, ionized wind from the central star has 'broken out' of the main nebula and is energizing filaments in the halo, injecting heat into them either by shock waves or more directly by fast particles from a $10^{8} \mathrm{~K}$ shocked stellar wind. Derived abundances might be incorrect.

The question is an important one, because if $\mathrm{N}, \mathrm{O}$ and $\mathrm{Ne}$ really can change their abundances relative to $\mathrm{H}$ by a factor 2 during the last $10^{5} \mathrm{yr}$ of AGB evolution, this would be an important new constraint on mixing and nucleosysnthesis in these stars. Manchado \& Pottasch also suggest that perhaps the nebular centres appear twice as metal-rich because hydrogen is deficient there. But a major objection to this is that $\mathrm{He} / \mathrm{H}$ ratios in PNs agree with those of local H II regions to within better than $10 \%$. 


\section{New Results for Magellanic Cloud Nebulae}

Studies of Cloud PNs are of special interest because the distances to both galaxies are known and the 'metallicities' of these systems are lower than in the Solar neighbourhood. Here then is a chance to study the results of stellar nucleosynthesis and mixing processes at different metallicities, by comparing abundances at these three sites. Previous work on Cloud PNs was reviewed by Barlow (1989).

I report here new results, including $\mathrm{C} / \mathrm{H}$ ratios, obtained in collaboration with N.A. Walton and M.J. Barlow. Preliminary results were given by Walton et al. (1990), and a discussion parallel to the present one is given by Barlow (1990). Our goals are to obtain an HR Diagram for Cloud central stars, and to measure nebular masses and abundances.

Data for 38 LMC and SMC nebulae were obtained both with the IUE, using new and archived spectra, and with the IPCS detector at the Anglo-Australian Telescope. The IPCS optical data replaces our less-accurate IDS data published by Monk et al. (1988). Standard methods were used for nebular analysis, with $\mathrm{T}_{e}$ from [O III] and [N II] line ratios and $\mathrm{N}_{e}$ from [O II] and [S II] ratios. A new set of ionization correction factors (icf's) were developed for the cases where certain ionic lines from the optical and IUE SWP camera were measured. The new icf's are based on the results of photo-ionization models for a sample of known (local) PN structures but computed with our mean Cloud abundances. The same grid of new models was used to predict the electron temperatures which should be adopted for each ionic stage. Ionized masses were found from absolute $\mathrm{H} \beta$ fluxes plus $\mathrm{N}_{e}$ values from forbidden-line ratios, together with adopted distances to the LMC and SMC of 47 and $57 \mathrm{kpc}$ respectively. Central star parameters were found from a very careful application of both Zanstra and Stoy methods. We distinguish between nebulae which should be the analogues of Galactic 'Type I' nebulae and others, using the criterion N/O > 0.5 for 'Type I' (really, N-rich) Cloud PNs.

Mean total ionized masses for a sample of $80 \mathrm{PNs}$ are $0.30( \pm 0.09)$ and $0.39( \pm 0.08) \mathrm{M}_{\odot}$ for SMC 'normal' and 'Type I', respectively; and $0.31( \pm 0.10)$ and $0.50( \pm 0.13) \mathrm{M}_{\odot}$ for the LMC. These refer to a sample picked as bright, easily-observable nebulae, and may thus be biased towards larger masses. The Type I ionized masses seem somewhat higher than the 'non-Type I's', but not at a statistically-significant level.

Abundances of $\mathrm{He}, \mathrm{C}, \mathrm{N}, \mathrm{O}$ and $\mathrm{Ne}$ for the sample of 38 bright nebulae are shown in Table 1. This also includes for comparison results for H II regions in each Cloud, taken from Dufour (1982) and Pagel et al. (1978). The He abundances are for an assumed $60 \%$ of the full corrections to HeI line fluxes for collisional effects (see 8. below).

Plots of abundances are shown in Figures 3-5. The major results are that:

1. Virtually all non-Type I PNs have $\mathrm{C} / \mathrm{O}>1$ (Fig. 3). This suggests that they have evolved from AGB carbon stars in each Cloud. Clegg (1990) compared the HR Diagrams of Cloud PNs and AGB stars in a distance-independent way, and found that indeed the central star luminosities match the upper end of the carbon star values, around $\mathrm{M}_{\mathrm{BOL}} \approx-$ 5.0.

2. Many Type I nebulae $(3 / 5$ in the $L M C$, and $3 / 3$ in the $S M C)$ have $C / O<1$. One interpretation of this is that their (massive) progenitors had very high mass-loss rates on the upper AGB, and in some cases AGB evolution terminated before enough ${ }^{12} \mathrm{C}$ was dredged up to give $\mathrm{C}>\mathrm{O}$. In this picture the high $\mathrm{N}$ abundances would arise from the 2 nd dredge-up. 


\section{TABLE 1. He, C, N, O, and Ne abundances}

MCPN: This work. H II regions: Pagel et al. (1978), Dufour et al. (1982)

Galactic PN: Torres-Peimbert \& Peimbert (1977), Aller \& Czyzak (1983)

Mean logarithmic abundances, $\mathrm{H}=12.0$

$\begin{array}{lllllll} & \text { He } & \text { C } & \text { N } & \text { C+N } & 0 & \text { Ne } \\ \text { SMC H II } & 10.91 \pm 0.02 & 7.16 \pm 0.04 & 6.46 \pm 0.12 & 7.24 \pm 0.05 & 8.02 \pm 0.08 & 7.22 \pm 0.12 \\ \text { Non Type I } & 11.01 \pm 0.04 & 8.87 \pm 0.27 & 7.44 \pm 0.17 & & 8.20 \pm 0.15 & 7.42 \pm 0.24 \\ \text { Type I } & 11.10 \pm 0.09 & 6.94 \pm 0.29 & \mathbf{7 . 7 7} \pm \mathbf{0 . 1 6} & & 7.61 \pm 0.13 & 7.12 \pm 0.08 \\ \text { All SMC PN } & 11.06 \pm 0.05 & 8.79 \pm 0.30 & 7.51 \pm 0.16 & & 8.15 \pm 0.16 & 7.38 \pm 0.24 \\ & & & & & & \\ \text { LMC H II } & 10.91 \pm 0.02 & 7.90 \pm 0.15 & 6.97 \pm 0.10 & 7.95 \pm 0.15 & 8.43 \pm 0.08 & 7.64 \pm 0.10 \\ \text { Non Type I } & 11.02 \pm 0.05 & 8.78 \pm 0.34 & 7.68 \pm 0.26 & & 8.41 \pm 0.18 & 7.61 \pm 0.25 \\ \text { Type I } & 11.10 \pm 0.08 & 8.45 \pm 0.50 & \mathbf{8 . 3 6} \pm 0.12 & & 8.41 \pm 0.20 & 7.80 \pm 0.19 \\ \text { LMC PN } & 11.04 \pm 0.06 & 8.70 \pm 0.35 & 7.98 \pm 0.24 & & 8.41 \pm 0.17 & 7.68 \pm 0.19 \\ & & & & & \\ \text { Gal PN } & 11.00 \pm 0.04 & & 7.96 \pm 0.32 & 8 \cdot 51 \pm 0.35 & 8.68 \pm 0.14 & 7.98 \pm 0.20 \\ \text { Gal H II } & 11.00 \pm 0.02 & & 7.57 \pm 0.33 & & 8.70 \pm 0.25 & 7.90 \pm 0.17 \\ \text { Solar } & 10.09 \pm 0.03 & 8.56 \pm 0.04 & 8.05 \pm 0.04 & & 8.93 \pm 0.03 & 8.09 \pm 0.10\end{array}$

Note: He abundances provisional, based on $60 \%$ corrn. (see text).

Figure 3 - Abundance Results for MC-PN (Walton et al. 1990)

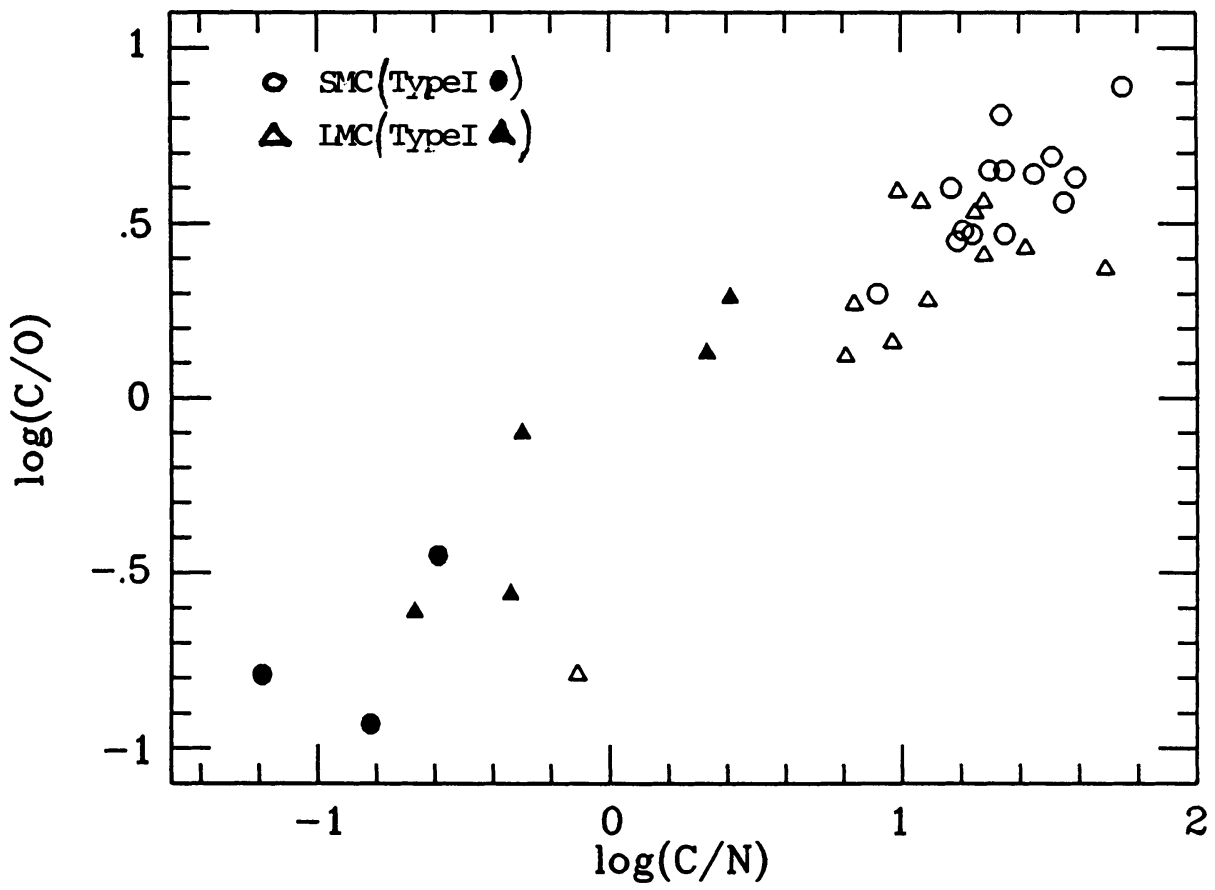




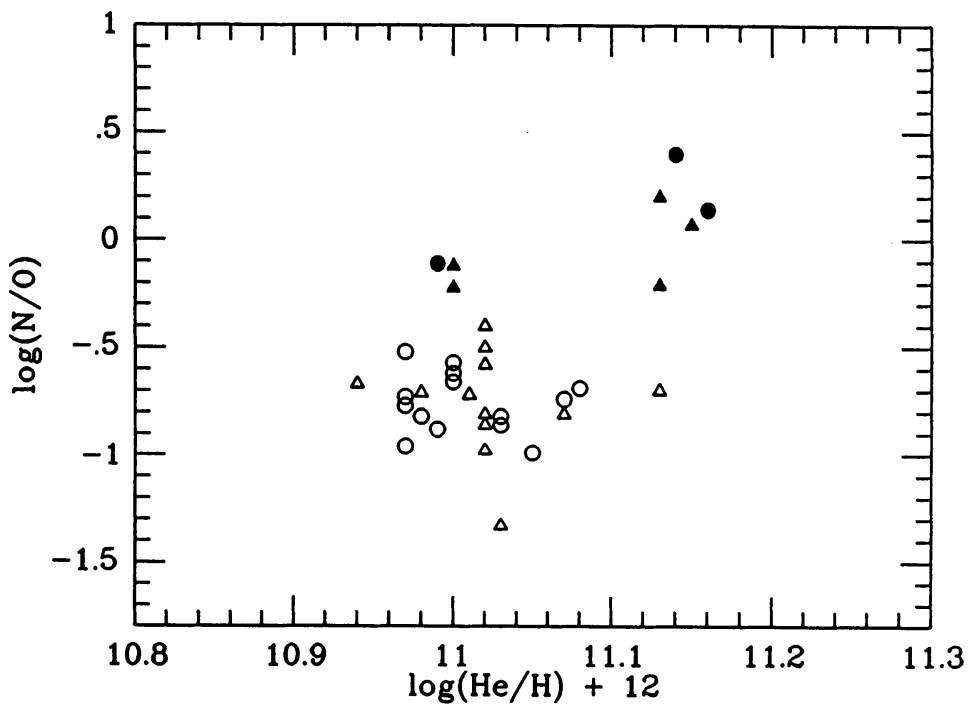

Figure 4. (N/O) versus $(\mathrm{He} / \mathrm{H})$ for Cloud PNs.

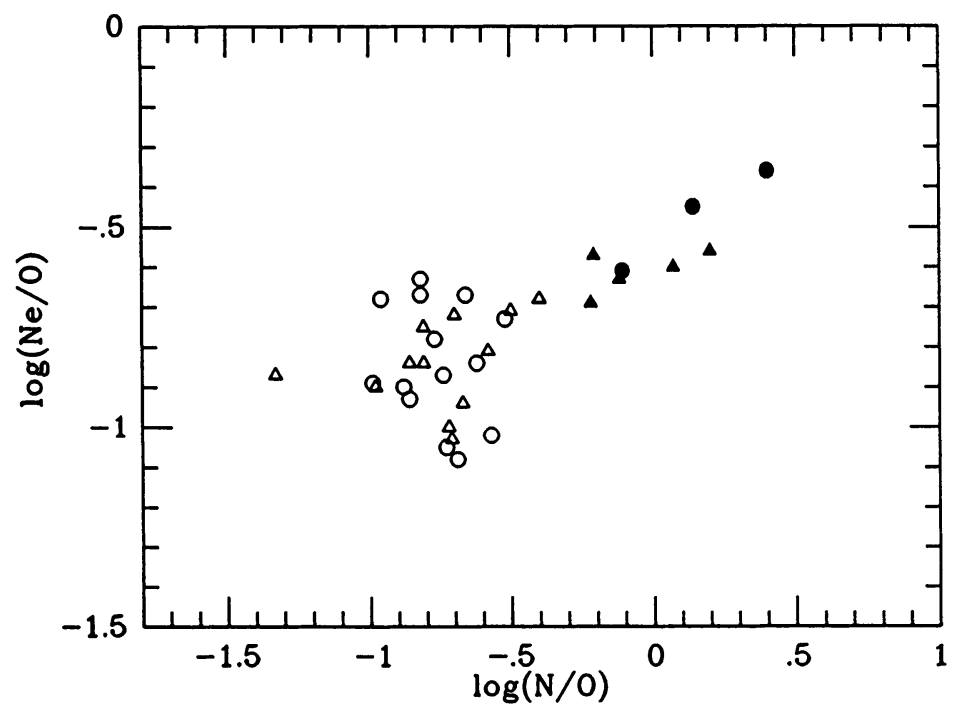

Figures5. ( $\mathrm{Ne} / \mathrm{O})$ versus $(\mathrm{N} / \mathrm{O})$. If the $\mathrm{Ne}$ excesses in Type I PN are real, they correlate with $\mathrm{N}$. 
In an alternative picture, the 3rd dredge-up did occur, but ${ }^{12} \mathrm{C}$ was in these cases mostly converted to ${ }^{14} \mathrm{~N}$ (with some ${ }^{13} \mathrm{C}$ ) during envelope burning on the upper AGB. Identification of $\mathrm{Li}$ and s-process enhancements in the (O-rich) upper-AGB stars (Lambert, these proceedings) would favour the second picture.

3. The correlation between $\mathrm{N} / \mathrm{O}$ and $\mathrm{He} / \mathrm{H}$ ratios (Fig. 4) shows much scatter, just as do such plots for local Galactic nebulae. The reasons, again, are surely as discussed in Sec. 2.1.

4. There is marginal evidence for higher Ne abundances in the LMC Type I nebulae (Fig. 5). This is also true for Galactic PNs. The most likely formation mechanism is $\alpha$-particle captures onto ${ }^{14} \mathrm{~N}$ yielding ${ }^{22} \mathrm{Ne}$ in the He-burning shell, followed by dredge-up. However, this result could be affected by systematic effects in adopted icf's for these (very highlyionized) nebulae, and further analysis is required to verify Ne overabundances.

5. There is a remarkable correlation (Fig. 3) extending over 3 decades between $\log (\mathrm{C} / \mathrm{O})$ and $\log (\mathrm{C} / \mathrm{N})$, with the range mostly provided by the Type I nebulae.

6. The three SMC Type I nebulae studied (N67, L305 \& L536) have low abundances of all heavy elements.

7. The mean mass, for 14 central stars analyzed, is $0.59( \pm 0.02) \mathrm{M}_{\odot}$ in both Clouds. This is for a sample of bright nebulae with nuclei still on the 'horizontal' (constant-luminosity) part of the evolutionary tracks. The selection effects are that more massive nuclei evolve rapidly on these tracks and are under-represented; and that some low-mass nuclei evolve so slowly that any nebula disperses before ionization, so they are not catalogued.

8. Helium abundances are similar to those of Galactic PNs. Unfortunately the uncertainty in corrections for collisional effects (see Sec. 2) gives uncertainty in the excess He over H II region values in each Cloud. Mean abundances for our whole sample, for 0,60 , and $100 \%$ of the theoretical collisional corrections to $\mathrm{HeI}$ lines are respectively $\mathrm{He} / \mathrm{H}=0.129,0.112$ and 0.104. The excess $\mathrm{He} / \mathrm{H}$ (PN minus $\mathrm{H}$ II regions) is 0.031 (SMC) \& 0.033 (LMC) for $60 \%$ correction, and $0.020 \& 0.026$ for $100 \%$ correction, with a typical uncertainty of \pm 0.015 .

9. Mean $\mathrm{C}$ abundances in SMC, LMC \& Galaxy are close. This actually implies a greater increase in $\mathrm{C} / \mathrm{H}$ in the $3 \mathrm{rd}$ dredge-up in Cloud $\mathrm{PN}$ if we assume (a) initial PN abundances equal to the relevant $\mathrm{H}$ II region values (b) efficiencies of $20,50 \& 100 \%$ for $\mathrm{C} \rightarrow \mathrm{N}$ conversion in the 1st dredge-up for SMC, LMC \& Galaxy, found by comparing initial $(\mathrm{C}+\mathrm{N})$ with final $\mathrm{N}$ abundance (Table 1). The 3rd dredge-up enhancements for the three systems are $\Delta(\mathrm{C} / \mathrm{H})=7.4 \times 10^{-4}, 5.6 \times 10^{-4}, \& 3.4 \times 10^{-4}$ respectively. So, production of carbon (measured as $\mathrm{C} / \mathrm{H}$ ) is larger in the Clouds, probably due to (a) lower metallicities - confirmation at lower-masses of predictions for higher mass stars (Becker \& Iben 1989, 1990) and perhaps also (b) different masses - because of 'bursts' of star formation, many 2-3 $\mathrm{M}_{\odot}$ stars may now be on the AGB in the Clouds (c.f. $1.5 \mathrm{M}_{\odot}$ in the Solar neighbourhood). [Note that this enhanced C-production is different from the 'titration experiment' effect whereby carbon stars are more easily produced when the $O$ abundance is low].

\section{References}

Aller, L.H. \& Czyzak, S.J. 1983. Ap. J. Suppl. 51, 211. Aller, L.H. \& Keyes, C.D. 1987. Ap. J. Suppl. 65, 405.

Barker, T. 1987. Ap. J. 322, 922. 
Barlow, M.J. 1989. IAU Symp. 131, ed. S. Torres-Peimbert, p. 319.

Barlow, M.J. 1990. Proc. IAU Symp. 148, in press.

Becker, S.A. \& Iben, I. 1979. Ap. J. $232,831$.

Becker, S.A. \& Iben, I. 1980. Ap. J. 237, 111.

Clegg, R.E.S. 1989. IAU Symp. 131, ed. S. Torres-Peimbert, p139.

Clegg, R.E.S. 1990. From Miras to Planetary Nebulae: Which Path for Stellar Evolution?, eds. A. Omont \& M-O. Mennessier (Editions Frontieres), p. 368.

Dinerstein, H.L., Lester, D.F. \& Werner, M. 1985. Ap. J. 291, 561.

Dufour, R.J. 1984. IAU Symp. 108, p. 353.

Harrington, J.P., Monk, D.J. \& Clegg, R.E.S. 1988. Ap. J. 231, 577.

Henry, R.B.C. 1990. Ap. J. 356, 229.

Henry, R.B.C., Leibert, J. \& Boroson, T.A. 1989. Ap. J. 339, 872.

Hoare, M.G. 1990. Mon. Not. R. Astr. Soc. 244, 193.

Hoare, M.G. \& Clegg, R.E.S. 1988. Mon. Not. R. Astr. Soc. 235, 1049.

Hollowell, D. \& Iben, I. 1989. Ap. J. 340, 966.

Iben, I. \& Renzini, A. 1983. Ann. Rev. Astr. Astrophys. 21, 271.

Iben, I. \& Truran, J.W. 1978. Ap. J. 220, 980.

Iben, I., Kaler, J.B., Truran, J.W. \& Renzini, A. 1983. Ap. J. 264, 605.

Jenkins, E.B. 1987. In Interstellar Processes, eds. D.J. Hollenbach \& H.A. Thronson.

Kaler, J.B. 1985. Ann. Rev. Astr. Astrophys. 23, 89.

Kaler, J.B. 1986. Ap. J. 308, 322.

Kaler, J.B. \& Jacoby, G.H. 1989. Ap. J. 345, 871.

Kaler, J.B. \& Jacoby, G.H. 1990. Preprint.

Kaler, J.B., Shaw, R.A. \& Kwitter, K.B. 1990. Ap. J. 359, 392.

Lambert, D.L., Gustafsson, B., Eriksson, K. \& Hinkle, K. 1986. Ap. J. Suppl. 62, 373.

Lattanzio, J. 1989. In Evolution of Peculiar Red Giants, eds. H. Johnson \& B. Zuckerman Manchado, A. \& Pottasch, S.R. 1989. Astron. Astrophys. 222, 219.

Middlemass, D., Clegg, R.E.S. \& Walsh, J.R. 1989. Mon. Not. R. Astr. Soc. $239,1$.

Middlemass, D., Clegg, R.E.S., Walsh, J.R. \& Harrington, J.P. 1990. Mon. Not. R. Astr. Soc. , submitted.

Monk, D.J., Barlow, M.J. \& Clegg, R.E.S. 1988. Mon. Not. R. Astr. Soc. 234, 583.

Peimbert, M. 1967. Ap. J. 150, 825.

Peimbert, M. 1978. IAU Symp. 76, ed. Y. Terzian, p. 215.

Peimbert, M. 1990. Reports on Progress in Physics, in press.

Peimbert, M. \& Torres-Peimbert, S., 1987a. Rev. Mex. Astr. Astrof., 14, 540.

Peimbert, M. \& Torres-Peimbert, S., 1987b. Rev. Mex. Astr. Astrof., 15, 117.

Renzini, A. \& Voli, M. 1981. Astron. Astrophys. 94, 175.

Rubin, R.H. 1969. Ap. J. 155, 841.

Scalo, J.M. 1981. In Physical Processes in Red Giants, eds. I. Iben \& A. Renzini, p. 77.

Smith, G.H. 1987. Publ. Astron. Soc. Pacific 99, 67.

Smith, V.V. \& Lambert, D.L. 1990. Ap. J. Suppl. 72, 387.

Stasinska, G. \& Tylenda, R. 1990. Astron. Astrophys. , in press.

Sweigert, A.V. \& Mengel, J.G. 1979. Ap. J. 229, 624.

Torres-Peimbert, S. \& Peimbert, M., 1977. Rev. Mex. Astr. Astrof., 2, 181.

Walton, N.A., Barlow, M.J., Monk, D.J. \& Clegg, R.E.S. 1990. IAU Symp 148, in press.

Wood, P.R. 1981. See Scalo 1981, page 205. 